\title{
Comparación entre la Clasificación AO pediátrica y la de Dias-Tachdjian en fracturas de tobillo infantiles
}

\author{
AGUSTINA PONZONE, ANDRÉS RONCORONI, FERNANDO MISCIONE, \\ EDUARDO L. BARONI y BIBIANA DELLO RUSSO
}

Hospital Nacional de Pediatría “Prof. Dr. Juan P. Garrahan”, Buenos Aires

\begin{abstract}
RESUMEN
Introducción: El objetivo del presente estudio fue analizar el grado de confiabilidad entre dos clasificaciones globalmente empleadas para fracturas de tobillo en la edad pediátrica.

Materiales y métodos: Se estudiaron 53 pacientes: 34 de sexo masculino y 19 de sexo femenino, con antecedentes de fractura de tobillo. Se emplearon dos clasificaciones. Se llevó a cabo un estudio detallado calculando el valor kappa de Cohen para la confiabilidad intraobservador y, para calcular el acuerdo interobservador, se dedujo el valor kappa utilizando el método de Fleiss.

Resultados: El acuerdo intraobservador e interobservador en las dos clasificaciones no fue convincentemente diferente entre los distintos grupos de examinadores.

Conclusiones: Durante la edad pediátrica, es posible encontrar diferentes tipos fracturarios y distintos mecanismos en una misma fractura. Se observó que estos patrones no quedaban englobados en ninguna de las dos clasificaciones, que fueron improductivas para la planificación quirúrgica.
\end{abstract}

Palabras Clave: Clasificación AO. Clasificación de Dias-Tachdjian. Fractura de tobillo. Comparación entre clasificaciones.

Comparison between the Pediatric

AO Classification and the Dias-Tachdjian

ClassifiCATION IN PEDIATRIC ANKLE FRACTURES

Recibido el 10-8-2012. Aceptado luego de la evaluación el 29-1-2013.

Correspondencia:

Dra. AGUSTINA PONZONE

agustina_ponzone@hotmail.com

\begin{abstract}
Background: The purpose of this study was to analyze the degree of reliability of the two most widely used classification systems for pediatric ankle fractures.

Methods: We studied 53 patients (34 boys and 19 girls) with ankle fractures. Two classifications were used. A detailed study was carried out calculating the Cohen's kappa index to measure intraobserver reliability and the kappa value was deduced using the Fleiss method to determine interobserver agreement.

Results: The intraobserver and interobserver agreement was not significantly different between the different groups of assessors.

Conclusions: In the pediatric age, different types of fractures and different mechanisms within an isolated fracture can be found. We found that many of these fracture patterns do not fit in any of the two classifications, which are unproductive to guide treatment selection.
\end{abstract}

\section{KEY WORDS: AO Classification. Dias-Tachdjian Classification. Ankle fracture. Comparison between classifications.}

\section{Introducción}

Una clasificación ideal para fracturas debe proveer información sobre la estabilidad de la fractura y el mecanismo de producción, pero además, debe tener un alto grado de reproducibilidad y deberá proporcionar una guía para la elección del tratamiento.

En la actualidad, no hay acuerdo en la interpretación, la evaluación y el tratamiento apropiados de las fracturas que comprometen la zona del tobillo en la edad puberal o prepuberal. 
Las fracturas de tobillo en niños representan el $11 \%$ de todas las fracturas, son unas de las fracturas más frecuentes (después de las del extremo distal del radio).

Se ha comunicado que las lesiones fisarias distales de la tibia y el peroné representan entre el $25 \%$ y el $38 \%$ de todas las lesiones de la fisis. Aproximadamente el 5\% de las fracturas de tobillo involucran la fisis.

No se ha evaluado con anterioridad la confiabilidad de dos de los sistemas más comunes de clasificación.

La clasificación de Dias-Tachdjian (1978) es una reforma de los mecanismos instaurados por la clasificación de Lauge-Hansen en el adulto, que describe el mecanismo por el cual se produce la fractura y la posición del pie en ese momento. ${ }^{1}$

La reciente clasificación AO pediátrica toma en cuenta tanto el mecanismo de producción como la afectación del cartílago de crecimiento, está íntimamente relacionada con los esquemas de fracturas fisarias de la clasificación de Salter y Harris. 2,3

El objetivo de este trabajo fue evaluar la confiabilidad interobservador y la reproducibilidad intraobservador de dos de las clasificaciones más usadas para fracturas de tobillo en pacientes con inmadurez esquelética.

\section{Materiales y métodos}

Se seleccionaron 53 pacientes con diagnóstico de fractura de tobillo y que contaban con los correspondientes estudios radiográficos anteroposterior y de perfil, admitidos en nuestro Servicio, entre 2002 y 2009. ${ }^{4}$ La edad promedio de los pacientes fue de 13,2 años, 34 del sexo masculino y 19 del sexo femenino (menores de 16 años y con fisis abiertas). Todos contaban con radiografías de frente estricto y perfil para la evaluación y el posterior tratamiento.

Los grupos estaban formados por dos cirujanos pediátricos experimentados y tres becarios con especial interés en trauma, pertenecientes al Servicio de Ortopedia y Traumatología del Hospital "Prof. Dr. J. P. Garrahan". Se les solicitó clasificar independientemente todas las fracturas de tobillo de acuerdo con la clasificación de Dias-Tachdjian y la clasificación AO pediátrica. Todos los participantes habían sido instruidos previamente con ambas clasificaciones.

La clasificación de Dias-Tachdjian (Fig. 1) consiste en 5 subtipos según el mecanismo de producción: tipo I, supinacióninversión; tipo II, supinación-flexión plantar; tipo III, supinación-rotación externa; tipo IV, pronación-eversión-rotación externa; tipo V, fracturas transicionales (Tillaux y triplanares). ${ }^{5}$ La fractura es llamada transicional de la tibia distal, porque su presentación depende de la edad, pues solo ocurre en adolescentes que están en transición hacia la madurez esquelética, cuando el cierre de la tibia distal ha comenzado, el cual es asimétrico y progresivo. ${ }^{6}$ Estas lesiones, que se traducen en fracturas tipo III y IV de Salter-Harris, plantean potenciales inconvenientes, como el cierre parcial o completo de la placa de crecimiento, ${ }^{7}$ y pueden generar acortamiento del miembro afectado, deformidad angular del eje del tobillo o incongruencia de la superficie articular. ${ }^{8}$

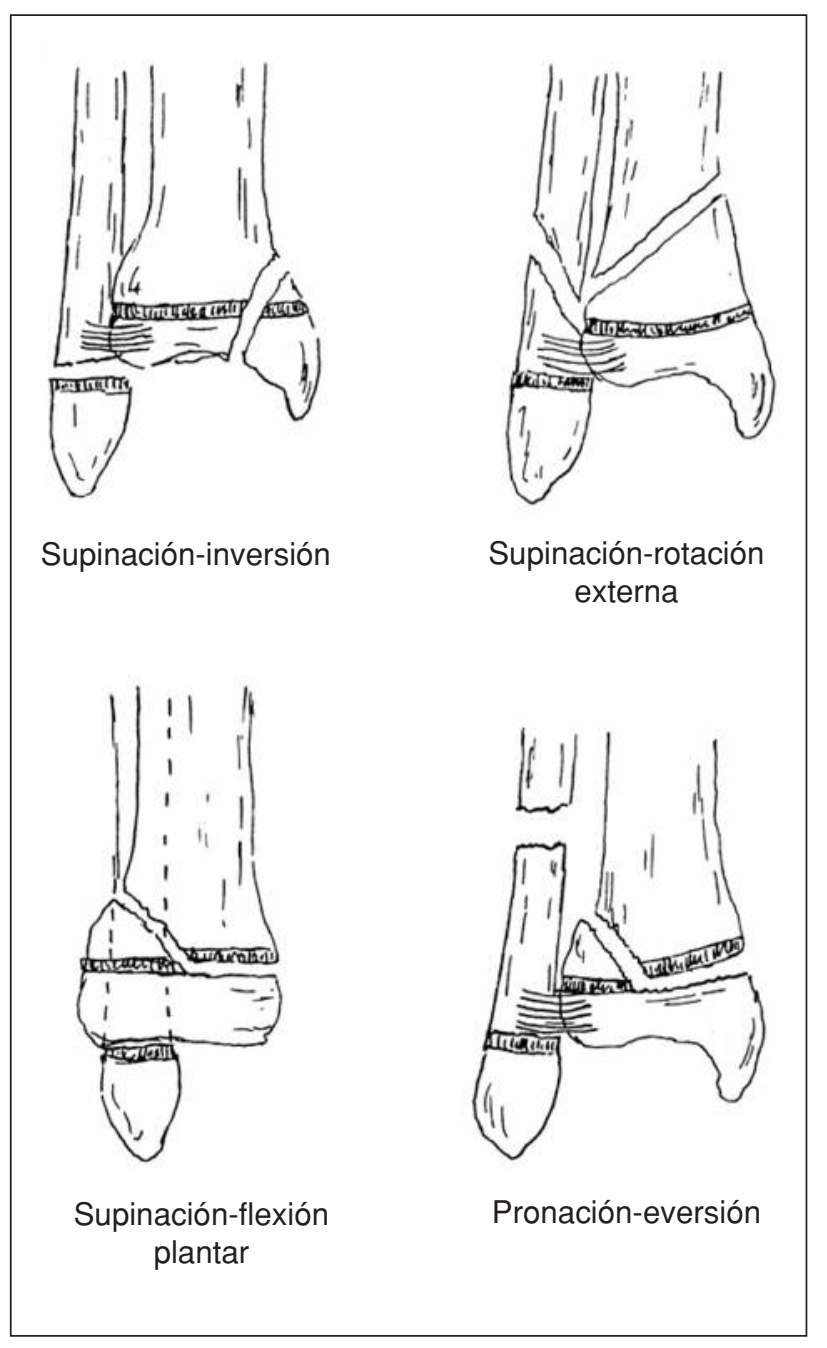

Figura 1. Clasificación de Dias-Tachdjian.

La clasificación AO pediátrica actual (Fig. 2) se basa en la clasificación de Müller-AO para adultos, pero se diferencia en que tiene en cuenta el cartílago de crecimiento y la división entre metáfisis y epífisis, y la divide en 3 grupos principales.

A los participantes se les otorgó la cantidad de tiempo necesaria para la evaluación, no estaban autorizados a discutir entre ellos sus resultados y no eran informados sobre la revaluación de las radiografías.

A los dos meses, cada participante debía revaluar las mismas radiografías en diferente orden, a las cuales se agregaba la evaluación del tipo de implante utilizado y la calidad de la reducción quirúrgica obtenida.

Se llevó a cabo un estudio estadístico calculando el valor kappa de Cohen para la confiabilidad intraobservador. A fin de calcular el acuerdo interobservador, se dedujo el valor kappa utilizando el método de Fleiss. ${ }^{9,10}$ Se interpretó el valor del coeficiente kappa de acuerdo con las guías propuestas por Landis y Koch: ${ }^{11}$ menos de 0,00: pobre exactitud; 0,00-0,20: leve exactitud; 0,21-0,40: razonable exactitud; 0,41-0,60: moderada exactitud; 0,61-0,80: considerable exactitud y 0,81-1,00: exactitud casi perfecta. 

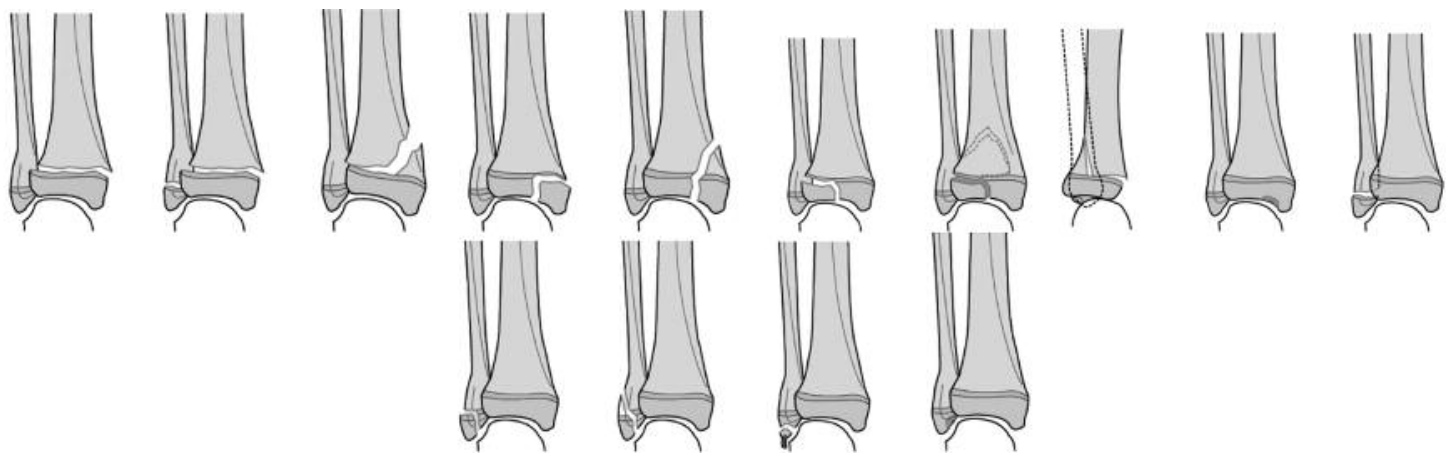

Figura 2. Clasificación AO pediátrica.

\section{Resultados}

En la Tabla 1, se muestran las fracturas clasificadas por los participantes usando la clasificación AO pediátrica y, en la Tabla 2, usando la de Dias-Tachdjian.

El acuerdo intraobservador e interobservador en las dos clasificaciones no fue significativamente diferente entre los cirujanos experimentados y los becarios.

El valor kappa interobservador entre cirujanos y becarios fue de 0,90 para la clasificación de Dias-Tachdjian, y de 0,65 para la clasificación AO pediátrica en la primera evaluación. En la segunda sesión, el valor kappa fue de 1 y 0,76 , respectivamente.

El valor del coeficiente kappa para la elección del implante utilizado fue de 0,48 para los cirujanos y de 0,38 para los becarios. El coeficiente kappa para la reducción obtenida en el posoperatorio fue de 0,84 para los cirujanos y de 0,75 para los becarios.

\section{Discusión}

En este estudio, se comparó la confiabilidad de dos clasificaciones muy usadas para las fracturas de tobillo: la clasificación de Dias-Tachdjian y la clasificación AO pediátrica. Se encontró una casi perfecta confiabilidad en la reproducción de la clasificación de Dias-Tachdjian y una considerable exactitud en cuanto a la clasificación AO.

Si bien hubo muy buena fidelidad a la hora de la reproducción de las clasificaciones, los participantes tuvieron problemas para la reproducción en varios puntos.

En cuanto a la clasificación de Dias-Tachdjian, la mayor cantidad de fracturas quedaron englobadas en el mismo subtipo (tipo I), que eran muy dispares en cuanto a los trazos fracturarios, el implante y la técnica quirúrgica por implementar. ${ }^{12}$
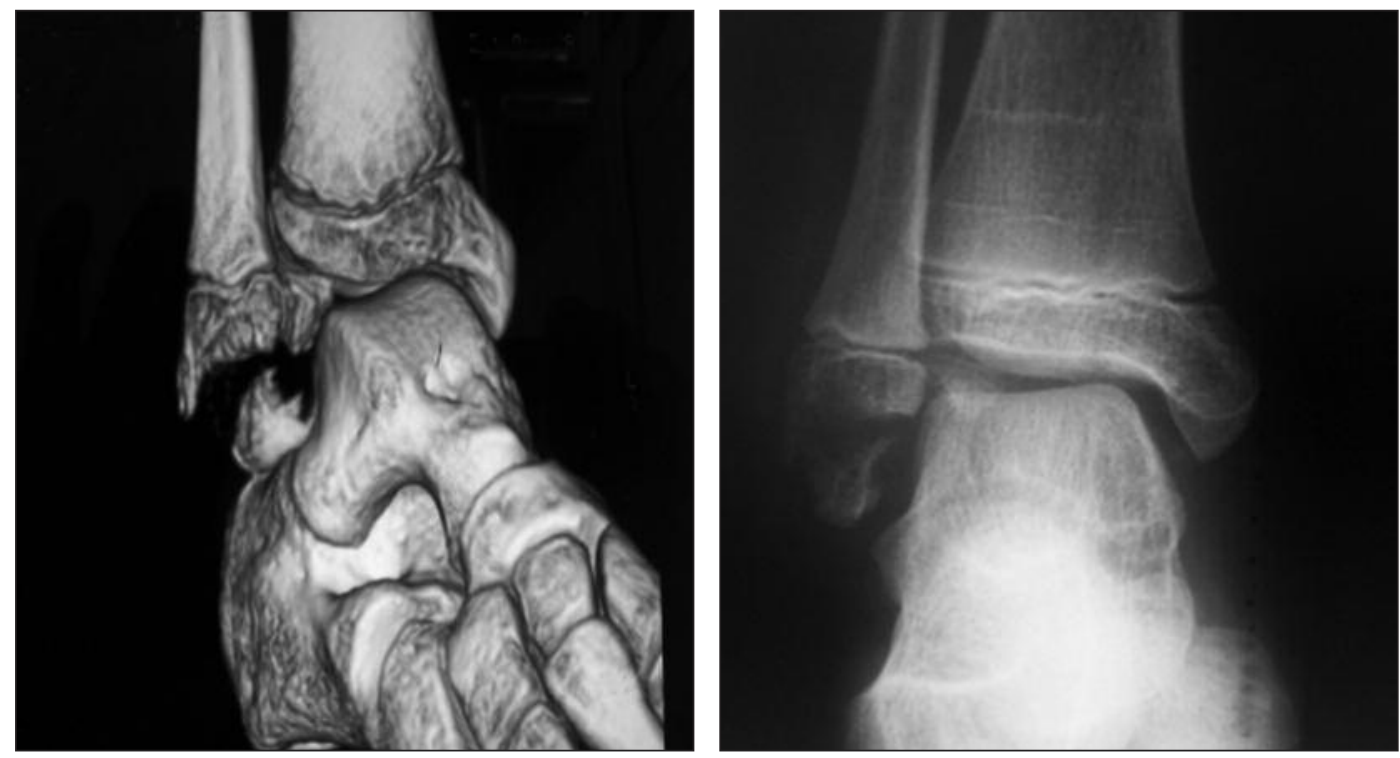

Figura 3. Fractura multifragmentaria de la epífisis del peroné. 

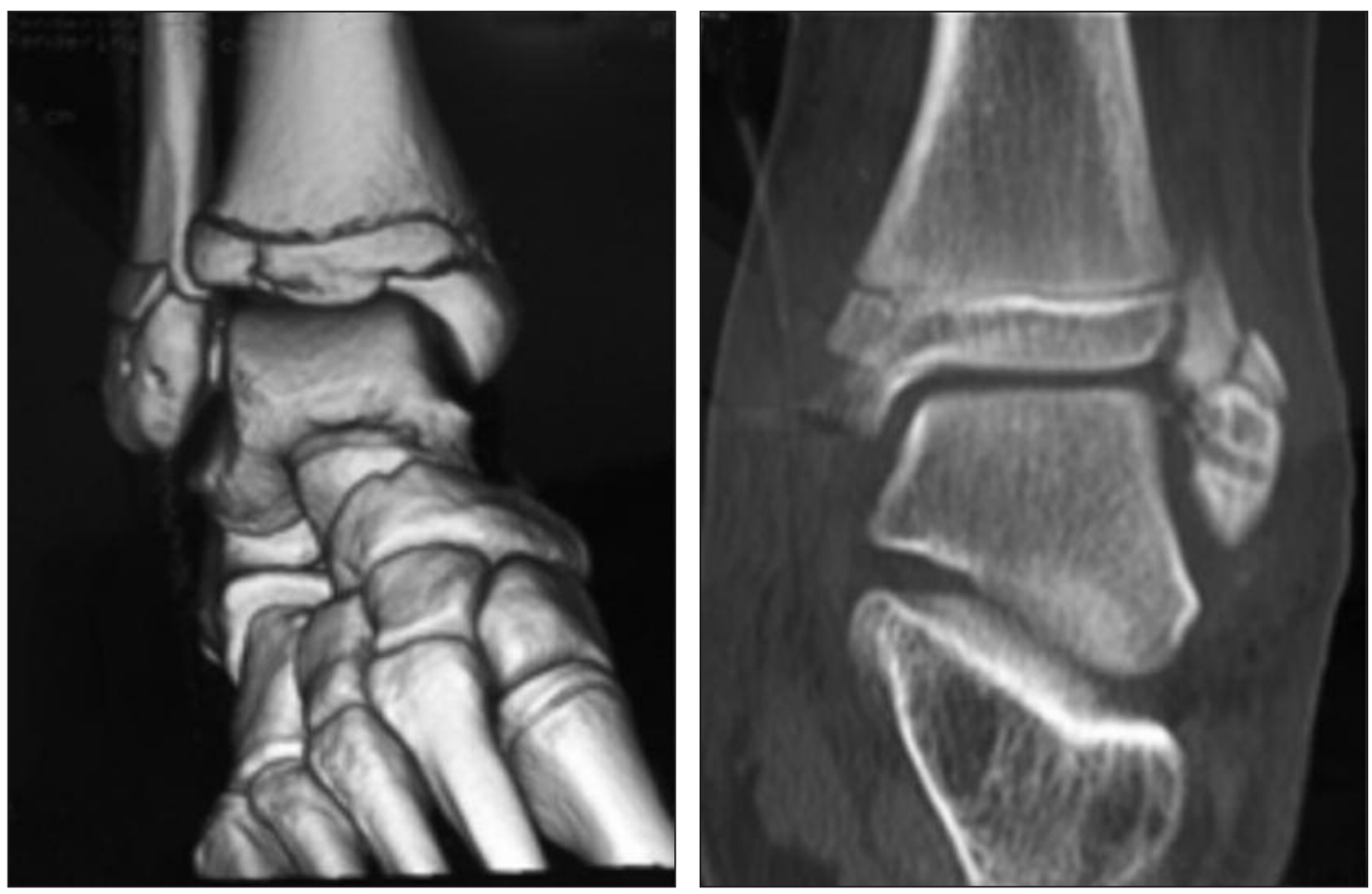

Figura 4. Fractura multifragmentaria del maléolo del peroné más fractura fisaria de la tibia, observada en los cortes tomográficos.

Tabla 1. Número de pacientes según la clasificación AO

\begin{tabular}{|l|c|c|}
\hline \multicolumn{1}{|c|}{ AO } & Becarios & Cirujanos \\
\hline $43 \mathrm{E} / 3.1$ & 18 & 13 \\
\hline $43 \mathrm{E} / 4.1$ & 3 & 3 \\
\hline $43 \mathrm{E} / 2.1$ & 5 & 5 \\
\hline $43 \mathrm{~T}-\mathrm{E} / 6.1$ & 8 & 8 \\
\hline $43 \mathrm{~T}-\mathrm{E} / 5.1$ & 8 & 5 \\
\hline $43 \mathrm{~T}-\mathrm{E} / 4.1$ & 3 & 3 \\
\hline $43 \mathrm{~T}-\mathrm{E} / 3.1$ & 5 & 10 \\
\hline $43 \mathrm{~T}-\mathrm{E} / 2.1$ & 0 & 3 \\
\hline $43 \mathrm{~F}-\mathrm{E} / 7$ & 0 & 3 \\
\hline $43 \mathrm{~F}-\mathrm{E} / 3.1$ & 3 & 0 \\
\hline Total & 53 & 53 \\
\hline
\end{tabular}

Con respecto a la clasificación $\mathrm{AO}$, el mayor número de dificultades se produjo debido a su alta complejidad y a la necesidad de un alto entrenamiento para su utilización.

Como es sabido, en los niños, se pueden encontrar diferentes tipos de trazos fracturarios y disímiles mecanismos en un mismo paciente; ${ }^{13}$ en dos casos, se obser-
Tabla 2. Número de pacientes según la clasificación de Dias-Tachdjian

\begin{tabular}{|l|c|c|}
\hline \multicolumn{1}{|c|}{ D-T } & Becarios & Cirujanos \\
\hline Tipo I & 29 & 29 \\
\hline Tipo II & 0 & 0 \\
\hline Tipo III & 0 & 0 \\
\hline Tipo IV & 8 & 8 \\
\hline Tipo V & 16 & 16 \\
\hline Total & 53 & 53 \\
\hline
\end{tabular}

vó que los trazos fracturarios eran multifragmentarios en unos y siempre combinados con trazos en los dos componentes óseos (tibia y peroné) y fueron evaluados por los clasificadores como trazos tipo 43F-E/3.1 (calificación $\mathrm{AO}$ para trazos que comprometen solamente el peroné) (Figs. 3 y 4 ).

Se arribó a estas conclusiones utilizando tomografías axiales con reconstrucción articular, técnica muy útil en los casos donde se presentan trazos multifragmentarios.

$\mathrm{Si}$ bien la clasificación AO da mejores pautas, ninguna de las dos clasificaciones proporciona ejemplos claros para el tratamiento de las fracturas. 


\section{Conclusión}

Es posible utilizar las dos clasificaciones en las diferentes fracturas pediátricas, la clasificación $\mathrm{AO}$ es más completa, pero ninguna nos guía para la planificación quirúrgica, por lo que resulta fundamental realizar un buen estudio por imágenes para definir la estrategia de tratamiento.

\section{Bibliografía}

1. Dias LS, Tachdjian MO. Physeal injuries of the ankle in children: classification. Clin Orthop Relat Res 1978;136:230-3.

2. Slongo TF, Audigé L. Fracture and dislocation classification compendium for children: The AO Pediatric. J Orthop Trauma 2007;21(Suppl. 10):S135-60.

3. Marsh JL, Slongo TF, Agel J, Broderick JS, Creevey W, DeCoster TA, et al. Fracture and dislocation classification compendium - 2007: Orthopaedic Trauma Association classification, database and outcomes committee. J Orthop Trauma 2007;21(Suppl. 10):S1-133.

4. Michelson JD, Ahn U, Magid D. Economic analysis of roentgenogram use in the closed treatment of stable ankle fractures. $J$ Trauma Inj Infect Crit Care 1995;39:1119-22.

5. Schnetzler KA, Hoernschemeyer D. The pediatric triplane ankle fracture. J Am Acad Orthop Surg 2007;15(12):738-47.

6. Harris EJ. Epiphyseal plate injuries in pediatric ankle traumatology. J Foot Surg 1981;20(3):145-7.

7. Cottalorda J, Béranger V, Louahem D, Camilleri JP, Launay F, Diméglio A, et al. Salter-Harris type III and IV medial malleolar fractures: growth arrest: is it a fate? A retrospective study of 48 cases with open reduction. J Pediatr Orthop 2008;28(6):652-5.

8. Leary JT, Handling M, Talerico M, Yong L, Bowe JA. Physeal fractures of the distal tibia. Predictive factors of premature physeal closure and growth arrest. J Pediatr Orthop 2009;29(4):356-61.

9. Cohen J. A coefficient of agreement for nominal scales. Educational and Psychological Measurement 1960;20:37-46.

10. Cerda J, Villarroel L. Evaluación de la concordancia interobservador en investigación pediátrica: Coeficiente de Kappa. Rev Chil Pediatr 2008;79(1):54-8.

11. Landis J, Koch G. The measurement of observer agreement for categorical data. Biometrics 1977;33:159-74.

12. Charlton M, Costello R, Mooney JF, Podeszwa DA. Ankle joint biomechanics following transepiphyseal screw fixation of the distal tibia. J Pediatr Orthop 2005;25:635-40.

13. De Sanctis N, Della Corte S, Pempinello C. Distal tibial and fibular epiphyseal fractures in children: prognostic criteria and long term results in 158 patients. J Pediatr Orthop 2000;9:40-4. 